\title{
Peer Support for Diabetes Management in Primary Care and Community Settings in Anhui Province, China
}

\author{
Xuefeng Zhong, MD, MPH, $\mathrm{PbD}^{1}$ \\ Zbimin Wang, MD, MPH \\ Edwin B. Fisher, $P b D^{2,3}$ \\ Chanuantong Tanasugarn, DrPH, \\ $M P H^{4}$
}

${ }^{1}$ Anhui Provincial Center for Disease Control and Prevention, Hefei, Anhui Province, China

${ }^{2}$ Department of Health Behavior, University of North Carolina at Chapel Hill

${ }^{3}$ Peers for Progress, American Academy of Family Physicians Foundation, Leawood, Kansas

${ }^{4}$ Department of Health Education and Behavioral Science, Faculty of Public Health, Mahidol University, Bangkok, Thailand

Conflicts of interest: Edwin B. Fisher, $P b D$, receives salary support for his role as Global Director of Peers for Progress from the American Academy of Family Physicians Foundation and has also received compensation for serving as a consultant and advisory board member for the Foundation for activities unrelated to the present paper.

\section{CORRESPONDING AUTHOR}

Edwin B. Fisher, PhD

Department of Health Behavior Gillings School of Global Public Health University of North Carolina-Chapel Hill Chapel Hill, NC 27599-7440

edfisher@unc.edu

\begin{abstract}
PURPOSE We evaluated a peer leader-support program (PLSP) for diabetes self-management in China in terms of acceptability and feasibility; implementation; perceived advantages; disadvantages and barriers; reach and recruitment; effectiveness in terms of diabetes knowledge and clinical impacts; adoption; and sustainability.
\end{abstract}

METHODS Within each of 3 cities in Anhui Province, 2 subcommunities were randomly assigned to usual care or PLSP. Peer leaders and staff of Community Health Service Centers (CHSCs) co-led biweekly educational meetings. Peer leaders also led biweekly discussion meetings, promoted regular care through the CHSCs, organized informal health promotion activities (eg, walking and tai chi groups), and provided informal individual support to participants through casual contact.

RESULTS Qualitative evaluations indicated acceptance of and positive responses to the program among patients, peer leaders, and CHSC staff. Implementation was successful in 2 of 3 subcommunities, the third failing for lack of staff resources. Reported advantages included peer support as a bridge between CHSCs and their patients. In 2 sites where the PLSP was implemented, analyses controlling for baseline differences and site showed significant benefits for PLSP relative to controls $(P<0.05)$ for knowledge, self-efficacy, BMI, systolic blood pressure, diastolic blood pressure, and both fasting and 2-hour post-prandial blood glucose. The Anhui Provincial Health Bureau has extended the PLSP model to other communities and to cardiovascular disease prevention and management.

CONCLUSION The PLSP was well accepted, feasible given sufficient administrative and staff resources, effective for those who participated, and generalizable to other sites and health problems.

Ann Fam Med 2015;13(Suppl_1):S50-S58. doi: 10.1370/afm.1799.

\section{INTRODUCTION}

The prevalence of diabetes mellitus among Chinese adults was $11.6 \%$ in 2010, or 113.9 million, and the prevalence of prediabetes was $50.1 \%$, or 493.4 million. ${ }^{1,2}$ Two-thirds of Chinese adults with diabetes have complications ${ }^{3}$ and only $11.5 \%$ exhibit satisfactory glycemic control. ${ }^{4}$ The Chinese health system, like many others, ${ }^{5}$ tends to emphasize clinical care but until recently has placed little emphasis on patient education.

A recent review of peer-support interventions documented contributions to basic health needs (eg, reducing childhood undernutrition), to primary care and health promotion, and to chronic disease management. ${ }^{6}$ Peer support can improve diabetes management ${ }^{7-12}$ but has not been adopted appreciably in China. An exception is a recent report of telephone peer support provided by a diabetes specialty service for patients of primary care physicians in Hong Kong. ${ }^{11}$ Peer support implemented through primary care is also a topic of growing interest. ${ }^{13-15}$

Our research project examined a peer-support intervention for type 2 diabetes in primary care community health services centers (CHSCs) in Anhui Province. Following models for implementation and dissemination research, ${ }^{16-18}$ it included initial formative evaluation of acceptability and 
feasibility and, at the end of the study period, assessment of advantages, disadvantages, barriers, reach, recruitment, effectiveness (within an evaluation subsample), and program sustainability and adoption.

\section{METHODS}

\section{Setting}

The Community Chronic Disease Management System provides population-wide primary care through CHSCs and their community health service stations (CHSSs). The government provides $¥ 25$ /person/year (in 2009-2010, approximately US\$3.50) to CHSCs to deliver a defined package of 11 basic public health services. For diabetes, these include a yearly clinical assessment $_{i}$ quarterly blood sugar assessment ${ }_{i}$ health education addressing healthy diet, physical activity, and medication adherence; and routine home follow-up visits. Traditionally, the CHSCs provide health education mainly through leaflets and brochures for patients with diabetes or through diabetes education presentations in community locations. These activities are coordinated through the Anhui Provincial Center for Disease Control and Prevention (CDC). Against this backdrop, the Peer Leader Support Program (PLSP) was implemented in 3 cities in Anhui.

An important feature of the setting was its integration of primary care with communities. Each community is subdivided into subcommunities, each with its own community-neighborhood committee and each served by its own CHSS. Individuals in a particular housing site receive their care through a clinical team assigned to that site. Figure 1, for example, shows the director of a CHSC pointing to a sign in the entrance of a residence indicating the names and telephone contact of the clinical team serving that building. Where the PLSP is implemented, peer leaders receive care through the same team as those with whom they live. Thus, as in Figure 2, peer leaders, their neighbors, and their clinical service team are connected so as to facilitate coherence of clinical care, peer support, and community activity.

Ethical approval for this study was obtained from the Ethics Committee for Human Research, Faculty of Public Health, Mahidol University (Proof Number: MUPH 2010-079).

\section{Selection of Communities}

Stratified sampling was used to select 1 city randomly in each of the northern, southern, and middle regions of Anhui. A district was then selected within each city and, within each district, 1 community of about 20,000 residents was randomly selected (Figure 3 ). These were the Yang Guang Community of Tonglin, the Da Qin
Community of Bangbu, and the He Ye-di Community of Hefei. Two subcommunities with similar characteristics in terms of demographics, customs, and social norms were randomly chosen from each community. The 2 chosen subcommunities, each served by its own CHSS, were then randomly assigned to intervention and control conditions.

\section{Formative Evaluation}

Formative evaluation addressed the feasibility, adaptability, and acceptability of the program and its key features relative to community and organization policy. It was also intended to engage and empower local communities to be part of program development. Focus groups were held in 2 communities and individual interviews in all 3 communities. The interviews included the leaders of each of the 6 CHSSs, 2 in each community, that had agreed to participate in the study, along with the leader of the district health bureau and

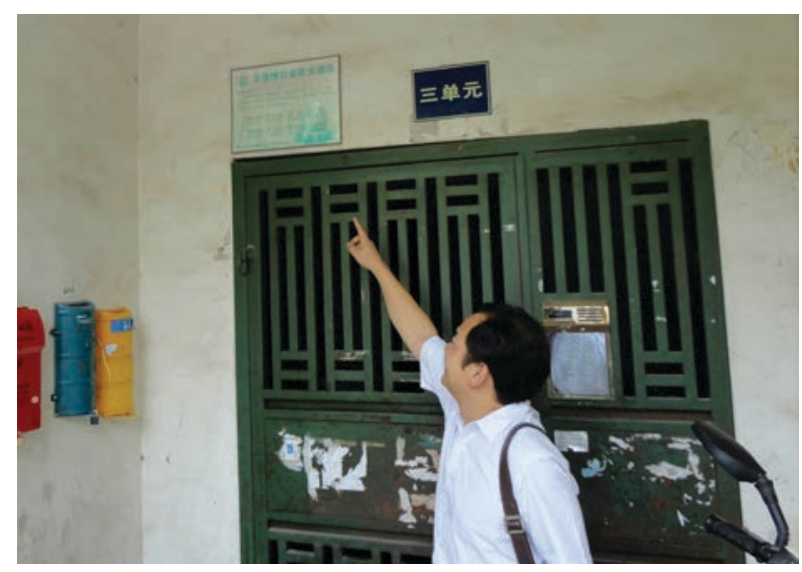

Figure 1. Community health center director pointing to a sign in a residence entryway that indicates the names and contact information for clinical team that serves residents of the building.

Figure 2. Relationships among peer leaders, clinical teams, and community residents in the Peer Leader Support Program.

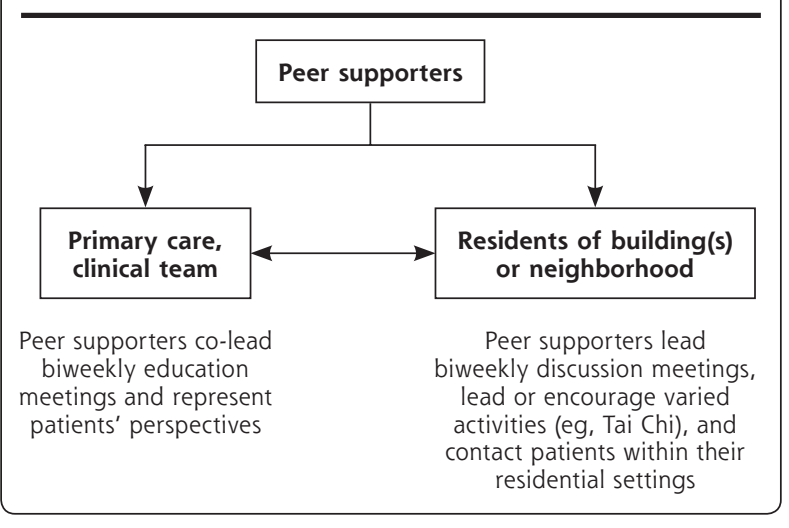


the leader of the neighborhood committee associated with each of the $6 \mathrm{CHSS}$.

Each focus group was conducted by 2 research team members. A third team member checked their notes for accuracy against audiotapes of the group meetings and participated in analysis. Content analyses identified the common concepts across sites and across roles. Response patterns and themes were similar for the 2 focus groups, so further groups were not pursued. Similar methods were used to evaluate implementation at the end of the study, also including interviews with patients and peer supporters.

\section{Implementation of the Peer Leader Support Program}

The formative evaluation indicated substantial support for the PLSP model and for a systematic study of its effectiveness. Therefore, the program was not altered before implementation.

\section{Peer Leader Selection and Training}

CHSC staff recruited 19 peer leaders who had been diagnosed with type 2 diabetes for more than 1 year, were willing to volunteer, and generally adhered to both medication and behavioral management regimens. Additional criteria were altruism, positive and sociable personality, availability of time, an understanding of the importance of patient confidentiality, good relationships with community residents, and leadership in their communities. Further selection was based on willingness to liaise with CHSC staff in response to unanticipated problems, to commit to the project schedule, to take on the responsibilities of peer leaders and adhere to program policies, to attend 3 days' training, and to contact group members frequently. Peer leaders were retired adults who had had diabetes for a mean of 9.3 years. Although some were nonprofessionals, a number had work experience in teaching, nursing, or the like. Sixteen of 19 were male (84.2\%).

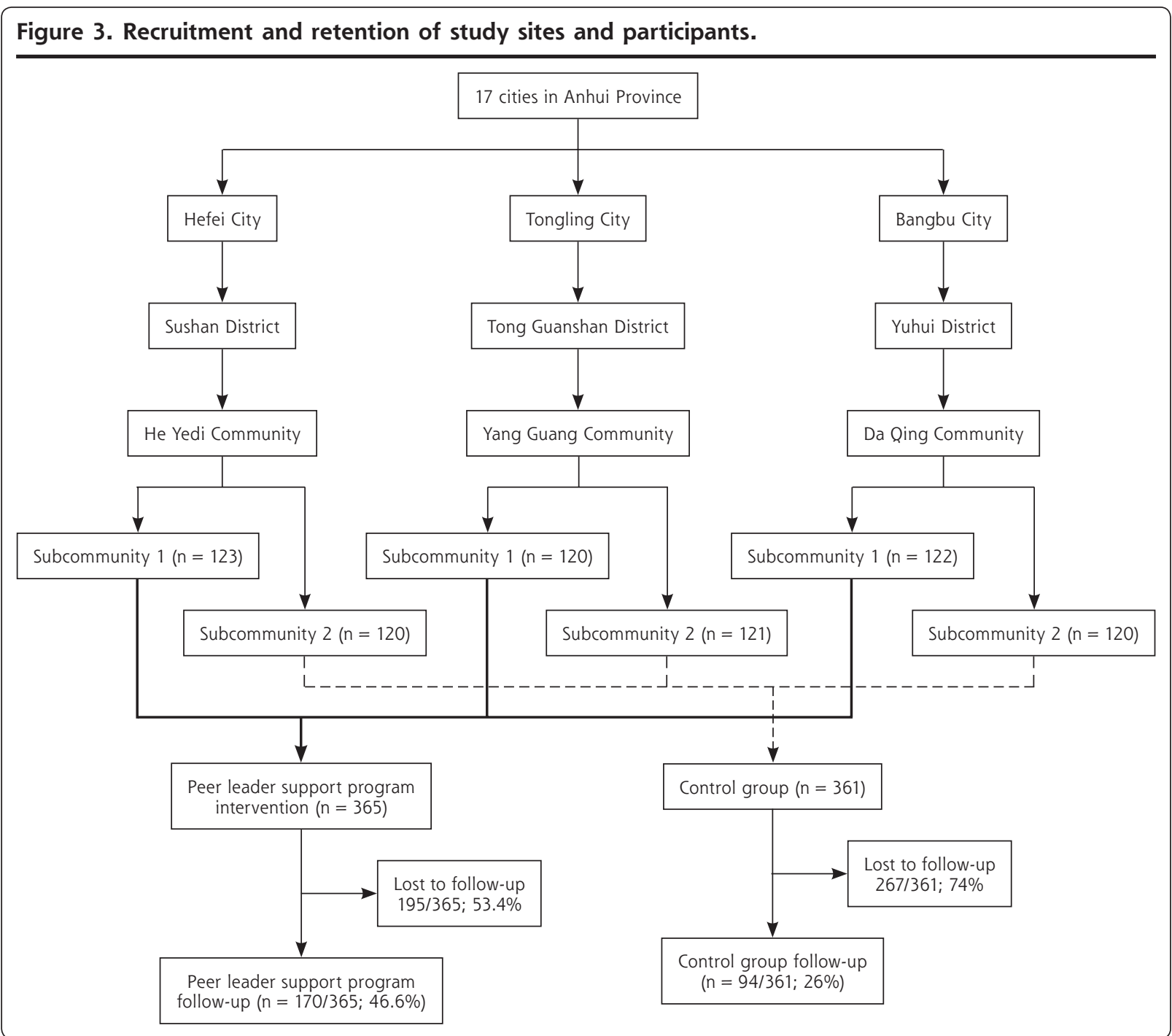


The Anhui CDC research team provided 3 days' training for the peer leaders, including an introduction to the PLSP and training in basic skills and diabetes selfmanagement. Training emphasized the key functions of peer support promoted by Peers for Progress: $:^{19,20}$

- Assisting and encouraging daily diabetes management

- Providing social and emotional support

- Linking with community resources and primary care at the CHSSs

- Providing ongoing support

Peer leaders were told how to seek support from their CHSS staff, CDC professionals, and tertiary hospital specialists if needed. They also met with each other bimonthly to share their experiences and exchange lessons. A peer leader handbook included materials for use with participants. CHSC staff also received training in the PLSP.

\section{PLSP Meetings and Activities}

Nineteen "peer support groups," 1 for each peer leader, were set up in the subcommunities randomized to the PLSP condition. Each group consisted of 10-15 participants. The protocol called for twelve biweekly education meetings over 6 months to be co-led by peer leaders with CHSC staff involvement titrated to peer leaders' needs. Meetings lasted 1.5 to 2 hours and covered a range of topics such as diet, physical activity, medications, foot care, stress management and depression, barriers to self-management, and obtaining resources and support from the community, family, friends, and the health system. For efficiency, groups were often combined, resulting in meetings of more than 30 participants and consequently limited opportunity for discussion. Accordingly, the protocol also called for peer leaders to lead 12 biweekly discussion meetings over 6 months. These reviewed the topics of the education meetings and included sharing experiences and modeling self-management practices.

Peer leaders also led or encouraged informal activities (for instance, walking and tai chi groups) among group members. Because peer leaders and participants lived within the same subcommunities, casual interactions and activities were common.

\section{Recruitment}

Participants were identified through a Community Chronic Disease Management System in each CHSC. We used the following inclusion criteria: (1) a diagnosis of type 2 diabetes made at a hospital, based on WHO criteria $^{21}{ }_{i}$ (2) age at least 15 years ${ }_{i}$ (3) residence in 1 of the project communities for more than 1 year. Exclusion criteria included inability to participate due to physical or mental disabilities.
CHSC staff contacted eligible patients by phone to explain the purpose of the study and details of the PLSP. Those providing consent were invited to visit their CHSS to provide baseline self-report and biophysical data. Generally, CHSS staff conducted face-to-face interviews for self-reported data because most participants had little education or were illiterate. Some interviews were also conducted by staff in participants' homes.

\section{Evaluation}

Implementation was monitored through research team records and work report forms of the CHSCs. Perceived advantages, disadvantages, and barriers to implementation were assessed through focus groups and interviews with community and clinic leaders, peer supporters, and patients at the conclusion of the PLSP. Assessment of sustainability and adoption was based on research team and CHSC records and reports.

\section{Effectiveness}

After community needs assessments and participant recruitment in the 6 subcommunities, baseline collection of clinical and self-report data took place over a 6-month period, from June to December, 2009. The intervention ran from January to July, 2010. Due to a public health emergency between August and October, follow-up data collection was delayed to October through December, 2010.

Evaluation included measures of diabetes knowledge, self-management practices, attitudes toward self-management, self-efficacy, and social support, as detailed in Supplemental Appendix 1 (http://annfammed.org/content/13/Suppl_1/S50/suppl/DC1). The measures were developed for this study. They were pilot tested in a subset of 35 adults with diabetes recruited from a CHSC outside the study communities. Internal consistency (Cronbach's $\alpha$ ) was 0.877 for diabetes knowledge, 0.815 for self-management practices, 0.842 for attitudes, 0.861 for self-efficacy, and 0.811 for social support.

\section{Statistical Analysis}

Analyses compared sites at baseline as well as participants who completed and did not complete follow up. Because patients received services from several peer leaders in each community, analyses did not include nesting of patients within peer leaders. Evaluation of outcomes controlled for site, age, education, income, and sex. With the sample size of 135 PLSP participants completing baseline and follow-up assessments (explained below), power was 0.77 ( 2 -sided, $\alpha=.05$ ) to detect a difference in fasting blood sugar of 0.5 $\mathrm{mmol} / \mathrm{L}(9.01 \mathrm{mg} / \mathrm{dL})$ between baseline and follow-up. 
Analysis was performed with SPSS, Version 19 (IBM) using the general linear model procedure (GLM).

\section{RESULTS}

\section{Acceptability and Feasibility}

As detailed in Table 1, key representatives from local Community Neighborhood Committees indicated that PLSP would be acceptable and feasible for their neighborhoods. Responses of CHSC directors, staff, and patients were also positive and included expressions of desire for "more training and direction" along with some concern among staff that "...the program may bring a large work burden for us." Accordingly, health authorities in the 3 cities agreed to provide policy, technical, and modest financial support to the PLSP.

\section{Implementation}

Table 2 includes, for each subcommunity, the average numbers of educational and discussion meetings held as well as the numbers of participants enrolled and participating. With only 1 CHSC staff member assigned to manage the program and co-lead group

Table 1. Observations from Participants, Peer Leaders, and Clinical Staff and Administrators Regarding Acceptability and Feasibility of the Peer Leader Support Program (PLSP)

"We will provide meeting rooms or other instrumental support if this project is implemented in our community." (Representative of Community Neighborhood Committee)

Community residents "are familiar with each other and like group activities such as Mahjong, dancing, walking and chatting...." (Representative of Community Neighborhood Committee)

"Peer leaders are like the bridge to link our CHSC with patients in their served neighborhood." (Community Health Center Director)

"This is an innovative program from which we could learn new knowledge and skills. Also, it may help us to use this approach for other health issues and diseases." (Community Health Center Director)

"We hope the Anhui CDC will give us more training and direction during project implementation." (Community Health Center Director)

"This project can help us to manage our registered patients with diabetes." (Community Health Center Station Staff Member).

"We don't have many intervention services from our CHSC." (Patient)

"If the community organized a diabetes peer support group, we would be keen to attend this group because this is beneficial for our disease and health." (Patient) meetings, implementation in community 3 did not achieve protocol objectives. Only 3 peer leaders were recruited and only 3 peer groups of 10 to 15 participants were organized. As a result, most participants from community 3 did not have the opportunity to attend group meeting and activities.

\section{Reach and Recruitment}

Women made up $49 \%$ of participants at baseline, with no difference by community or intervention. GLM analyses evaluated differences in baseline characteristics by condition (PLSP vs control), community (1,2 and 3 ), and the interactions between condition and community. (Supplemental Appendix 1 at http://annfammed.org/ content/13/Suppl_1/S50/suppl/DC1.) We found a significant interaction between communities and condition (PLSP vs control) for age and significant differences by condition for education and income. Accordingly, age, education, and income were included as control variables in all statistical evaluations.

Figure 3 shows the flow of 726 participants through the study, 365 in the intervention and 361 in the control subcommunities. Because of the CHSCs' responsibilities to their communities, PLSP was open to all individuals with diabetes, a number of whom began participating after the program began. As a result, 726 individuals completed baseline survey and $533 \mathrm{com}$ pleted follow-up surveys. Reasons for loss to follow-up included moving away, ceasing to participate in the program and its evaluation because of lack of time or health problems that interfered, and loss of interest.

Of the 365 individuals in the PLSP study population, $279(76.4 \%)$ attended at least 6 meetings and activities. One hundred and seventy completed both baseline and follow-up and attended 10 or more meetings or group activities. Among controls, 94 completed baseline and follow-up.

Because of the poor implementation in community 3 and its failure to recruit any participants for follow-up in its control subcommunity, it was dropped from further analyses. This resulted in a total of 135 PLSP and 94 control participants from communities 1 and 2 who completed follow-up and were therefore included in the evaluation of changes in study variables. Among those

Table 2. Average Numbers of Key Meetings and Activities and of Participants in Each PLSP Subcommunity

\begin{tabular}{lccc}
\hline Category & Subcommunity $\mathbf{1}$ & Subcommunity $\mathbf{2}$ & Subcommunity $\mathbf{3}$ \\
\hline $\begin{array}{l}\text { Average number of biweekly educational meetings co-led } \\
\text { by CHSC staff and peer leaders (12 by protocol) }\end{array}$ & 8.78 & 7.73 & 4.24 \\
$\begin{array}{l}\text { Number of biweekly discussion meetings led by peer } \\
\text { leaders (12 by protocol) }\end{array}$ & 11.63 & 9.35 & 6.47 \\
Number of enrollees & 123 & 120 & 122 \\
Number participants who attended $\geq 6$ meetings & 111 & 128 & 40 \\
\hline
\end{tabular}


Table 3. Changes in Outcome Variables Comparing PLSP and Control Participants Who Completed Follow-up

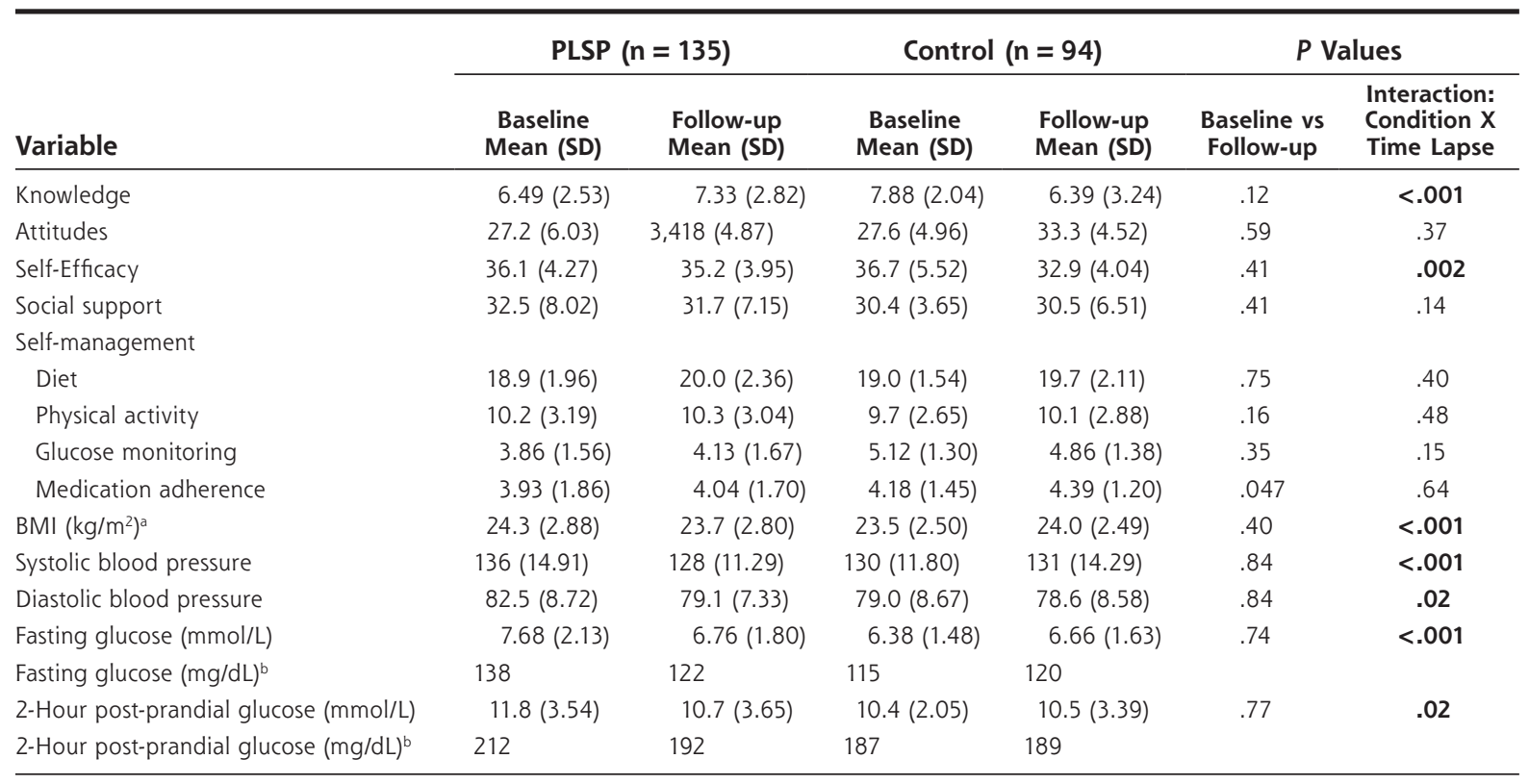

PLSP = peer leader support program.

Note: The table is derived from GLM repeated measures analyses controlling for sex, age, education, family income, and community.

\section{Description of Self-Report Measures}

Knowledge: Total of 12 items: 4 concerning glucose; 3 concerning diabetic complications; 2 concerning diet; and 3 concerning insulin. Correct responses received 1 point and incorrect answers or "don't know/unsure" received 0 , yielding a range of 0 to 12 . Higher scores indicate greater knowledge.

Attitudes (ie, attitudes toward self-management): 9 items scored 1 to 5 (strongly disagree to strongly agree), yielding a range of 9-45. Higher scores indicate more positive attitudes.

Self-efficacy: 9 items scored 1 to 5 (strongly disagree to strongly agree), yielding a range of 9-45. High scores indicate greater self-efficacy.

Social support (ie, perceived support for self-management): 9 items scored 1 to 5 (never to always), yielding range of $9-45$. High scores indicate greater perceived support.

Self-management: 9 items, scored 1 to 5 , resulting in subscores for diet (4 items, with a range of 4 to 20), physical activity (2 items, with a range of 2 to 10 ), glucose

monitoring ( 2 items, with a range of 2 to 10 ), medication adherence ( 1 item, with a range of 1 to 5$) .22$ Higher scores indicate better self-management practices.

a For groups of Chinese origin, BMI values of $24.0 \mathrm{~kg} / \mathrm{m}^{2}$ for men and $23.0 \mathrm{~kg} / \mathrm{m}^{2}$ for women have been recommended as cut-offs for obesity. ${ }^{23}$

bGlucose levels in $\mathrm{mg} / \mathrm{dL}$ are conversions from means expressed as $\mathrm{mmol} / \mathrm{L}$.

completing follow-up, $41 \%$ were women, in comparison to $55.3 \%$ of those not completing $(P=.002)$.

Supplemental Appendix 2 compares those completing and not completing follow up on other study variables among PLSP and control sites. Within the PLSP, those who completed follow-up had greater education, greater diabetes knowledge, more positive attitudes toward self-management, and greater BMI. There were several significant interactions between condition and community. While those in PLSP who completed follow-up had greater diabetes knowledge, control participants who completed follow-up had less. Also, those in PLSP who completed follow-up were more likely to report glucose monitoring, but control participants were less likely to do so. Control participants who completed follow-up had lower BMI in contrast to the greater BMI noted above for PLSP participants who completed follow-up. In summary, then, sex, age, education, and income were included as control variables in subsequent analyses of effectiveness. Knowledge of diabetes, attitudes toward self management, and BMI were not included as control variables because they were included as outcomes.

\section{Effectiveness}

Table 3 includes baseline and follow-up measures of diabetes knowledge, attitudes, self-management behaviors, and clinical outcomes for the PLSP and control participants who completed follow-up in Communities 1 and 2, controlling for sex, age, education, income, and community. Significant interactions between condition and time lapse from baseline to follow-up indicated greater favorable changes for PLSP participants for diabetes knowledge, self-efficacy, BMI, systolic and diastolic blood pressure, fasting glucose, and 2-hour post-prandial glucose. For example, PLSP participants showed a reduction in fasting glucose levels from 7.68 $\mathrm{mmol} / \mathrm{L}$ to $6.76 \mathrm{mmol} / \mathrm{L}(138 \mathrm{mg} / \mathrm{dL}$ to $121 \mathrm{mg} / \mathrm{dL})$ while those in the control communities showed a slight increase from $6.38 \mathrm{mmol} / \mathrm{L}$ to $6.66 \mathrm{mmol} / \mathrm{L}(115 \mathrm{mg} /$ $\mathrm{dL}$ to $120 \mathrm{mg} / \mathrm{dL})$. The difference between these 2 patterns was highly significant $(P<.001)$. 


\section{Perceived Advantages, Disadvantages, and Barriers to Implementation}

Qualitative evaluation with CHSC staff, peer supporters, and patients at the conclusion of PLSP identified barriers to and facilitators of implementation, these are detailed in Table 4. Barriers ranged from staff and financial resources within the CHSSs to inconvenient meeting times.

Most peer leaders believed that the PLSP benefited both the patients in their groups and themselves, viewed the PLSP as important, were happy with their work, and said they would like to remain peer leaders. Facilitating factors included assistance peer leaders reported receiving from CHSC staff, skills staff reported learning through the program, and 1 CHSC director's opinion that the PLSP improved provision of care. Staff also advocated greater duration of the PLSP. Participants noted no negative aspects of the PLSP but noted the value of both practical, specific assistance and emotional support it provided.

\section{SustainabilitylAdoption}

Based on these findings, the Anhui Provincial Health Bureau has expanded PLSP to other communities in the province and to cardiovascular disease prevention and management. This has been facilitated by national health reform, which has enabled the assignment of additional staff to disease management and prevention so that programs like PLSP can become part of routine work, not extra work.

\section{DISCUSSION}

To our knowledge, no previous research has examined the feasibility, acceptance, and benefits of peer support for diabetes management based in community primary care in China. The PLSP proved well accepted and feasible if it is provided administrative support and staff resources. It was effective in improving diabetes knowlover a longer time.

\section{Barriers for CHSCs} tion Staff Member) refreshments. (Peer Leader) edge and clinical outcomes for those who attended peer-support groups. Peer-led meetings and activities encouraged supportive networks among group members in their familiar neighborhoods in a way compatible with Chinese culture. The Anhui Provincial Health Bureau's extension of PLSP to other settings and health

\section{Table 4. Observations of Participants, Peer Leaders, and Clinical Staff and Administrators Regarding Implementation and Emergent Features of the Peer Leader Support Program (PLSP)}

Facilitating factors for the Community Health Support Centers (CHSC)

A CHSC director indicated that peer leaders can help CHSC staff implement primary care more effectively, for example through group monitoring and care, with peer leaders helping to invite patients to attend. Instead of telephoning patients individually, peer leaders can contact them in groups and through their neighborhoods.

CHSC staff suggested developing an ongoing network from the provincial level to community levels to facilitate program implementation, requiring that patients with diabetes receive diabetes self-management education, and improving PLSP outcomes through implementation

Limited time and human resources, system coordination, and external locus; "We worry a little bit that the program may bring a large work burden for us." (Community Health Center Sta-

Although required to provide public health services, CHSCs tended to focus on fee-producing clinical services, while public health services depended largely on local government.

Facilitating factors for peer leaders

Instrumental support for peer leaders through collaboration with CHCS staff: "We contacted CHSC staff and professionals more frequently.... I have more close ties with CHSC staff. If we want them to help us, they always can do that."

Confidence: Training, discussions, and participation in the PLSP had increased diabetes knowledge, skills for diabetes self-management, and confidence in leading group meetings/activities.

Sense of importance: PLSP meetings and activities were viewed as the best ways for those with diabetes to help each other. Peer leaders felt pride that they were able to meet needs, provide help, and be valuable to others and their communities.

Barriers for peer leaders

Need for modest financial support for materials (eg, table tennis balls), transportation, or

Limited ability to promote diabetes management; "I would like to have more time training."

"I would like to have a chance to learn from others. Could the CHSC organize us to visit another community to learn from them?" (Peer Leader)

Facilitating factors for participants

Most participants expressed positive views of group meetings and activities and valued the openness of their implementation, such as by being told in advance of topics to be discussed.

Barriers for participants

Reasons cited for not attending group meetings or activities included weather, family matters, inconvenient or non-preferred meeting times, and planned topics of little interest.

Practical, specific support provided by peer leaders

"...it is difficult to get up in the morning to do exercise. My peer leader had a phone call in the morning for weeks and waited for me under my building to get me doing morning exercise. Then I could get up every morning to do exercise with the group." (Participant)

"I often forgot the date to get my blood sugar checked free by the CHSC. My peer leader always gave me a call to mention that I needed to go to CHSC for a routine check the next day. If I still forgot, he would call me again to ask me to come to the CHSC for a check." (Participant)

Emergence of emotional support

"The peer support group is like our 'second home' in which you can say what you want to say, without worry about misunderstanding and discrimination." (Participant)

"We could easily talk with peer leaders and peer group members when we felt unhappy, and we didn't want to talk with our children because we didn't want to burden them." (Participant)

Dissemination of the PLSP model

CHSC leaders saw peer support as an innovative strategy for addressing needs other than diabetes management: "The peer support approach is a good method to help manage chronic disease, not only for diabetes... I am preparing to set up peer groups to target hypertension patients." (CHSC Director) 
problems attests to its promise as a broad strategy for improving chronic disease care and prevention.

Group activities are traditional in Chinese culture, especially for older adults. This may have enhanced participation in the informal groups based on shared interests-a jogging group, for example. Additionally, residents living in the same neighborhood are familiar with each other and generally help each other. Also of note, all peer leaders and most participants were older adults (average age $63 \pm 9.41$ years), and retired $(62.8 \%)$ providing time to attend group meetings and activities. Practical, specific support from the PLSP may have contributed to participants' increased self-efficacy. ${ }^{24}$

Success of the PLSP for diabetes and its extension to prevention and management of cardiovascular and other diseases also benefitted from recent policies of the national government encouraging the primary care CHSCs to serve as "health gatekeepers" and to manage referrals to specialist care and hospitals. ${ }^{25}$ This includes the government’s providing $¥ 25$ /person/year to CHSCs for implementing 11 primary care and preventive packages.

Emotional support emerged as a benefit of the PLSP. With cultural emphases on harmony and the interests of the family, relatives are obligated to support each other but, at the same time, reluctant to place a burden on their families. ${ }^{26}$ The PLSP apparently provided a setting in which individuals could discuss feelings without fear of obligating others.

Several aspects of the roles of peer leaders deserve attention. Co-leading educational meetings with CHSS staff might be thought to compromise the "peerness" of the peer leaders. They reported, however, that they valued not having to worry about making mistakes, but being able to focus on their roles as peers and the activities they promoted. This reflected a view of their role as distinct from and complementary to health professionals, not as "junior doctors."

Peer leaders reported good working relationships with CHSS staff, providing feedback to CHSS staff about group members' needs, questions raised, and needs for specialty referrals. Participants valued the peer leaders as "our" representative who could express "our" needs and requirements to CHSCs and tertiary hospital specialists. In this, peer leaders may offer a valued counterforce to the otherwise hierarchical nature of medical care. The PLSP also embodied community participation strategies needed for chronic disease prevention and control in the daily lives of individuals. ${ }^{27,28}$ This was valued by CHSC directors, 1 of whom noted that peer leaders may serve as "the bridge to link our CHSC with patients in their served neighborhood." This reflects broader discussion of contributions of peer support to primary care..$^{13-15}$
Men comprised $84 \%$ of peer leaders. Most women invited to become peer leaders felt that, although they were retired, housework and looking after grandchildren prevented their commitment to group meetings and activities. Also, most women in the generation sampled have lower education levels than their male counterparts.

Variability in implementation underscores the importance of organizational support to program implementation, issues just beginning to be studied in peer support..$^{15}$ The limited implementation in Community 3 also reflects transitions in which hospitals have become CHSCs but, in so doing, have needed new income sources. At the time of the study, the Community 3 CHSC focused on clinical income and also lacked public health professionals and experience, limiting its PLSP implementation. These transitions are now generally completed and the associated limitations less common. That some peer-leader activities varied from group to group (especially informal activities not specified in the protocol) raises issues concerning standardization and our achievement of treatment fidelity. The PLSP addressed this through standardization not around specific activities but around key functions of peer support (assistance in daily management, social and emotional support, linkage to clinical care and community resources, and ongoing availability of support). ${ }^{20,29}$

\section{Limitations}

Within this dissemination and implementation study, threats to validity and potential sources of bias included randomization of communities before collection of baseline data, collection of outcome data by community nurses not blinded to community allocation, conduct of focus groups and structured interviews by research staff, failure of analyses to include duration of diabetes and some measure of complications, and substantial numbers lost to follow-up.

Initiation of the PLSP may have motivated CHSC staff to provide better diabetes care. Monitoring of prescribing practices, however, did not indicate differences in delivery of routine diabetes care between intervention and control subcommunities.

The present results are consistent with other reports of benefits of peer support in diabetes management. ${ }^{7-12}$ Interestingly, the study of telephone peer support in Hong Kong ${ }^{11}$ failed to achieve incremental clinical benefit when added to comprehensive care that included coordination through a registry, initial patient education, and initial appraisals, quarterly reports and algorithm-based recommendations for care to patients and clinicians. ${ }^{30}$ Consonant with the present study's identification of the importance of emotional support, the Hong Kong peer-support program reduced emotional distress as well as hospitalizations among approx- 
imately $20 \%$ of participants who were initially above norms on measures of anxiety, depression and stress. ${ }^{11}$

To read or post commentaries in response to this article, see it online at http://www.annfammed.org/content/13/Suppl_1/S50.

Key words: peer support; community; diabetes

Submitted August 18, 2014; submitted, revised, March 13, 2015; accepted March 24, 2015.

Funding support: Funding for this research was provided by the American Academy of Family Physicians Foundation through the Peers for Progress program with support from the Eli Lilly and Company Foundation and by the Anhui Provincial Health Bureau.

Previous presentations: Reciprocal cooperation between peer supporters and community health centers in China. Zhong X. Annual Meeting of the Society of Behavioral Medicine; April 11 - 14, 2012; New Orleans, Louisiana. Peer support interventions for diabetes and community health centers in China. Zhong X. Biannual International Congress of Behavioral Medicine; August 29 - September 1, 2012; Budapest, Hungary.

Acknowledgments: We would like to thank the following organizations which supported this programs: Hefei City Health Bureau, Hefei City CDC, Shu-shan District CDC, Heyidi CHSC, Tongling City Health Bureau, Tongling City CDC, Tong guan-shan District CDC, Yangguan CHSC, Rendong CHSS, Bangbu City Health Bureau, Bangbu City CDC, Yuhui District CDC, Daqing CHSC.

We also thank Jennifer Butterworth Robinette, MA, who provided valuable assistance in preparing the manuscript.

Supplementary materials: Available at http://www.AnnFamMed. org/content/13/Suppl_1/S50/suppl/DC1/

\section{References}

1. Yang W, Lu J, Weng J, et al; China National Diabetes and Metabolic Disorders Study Group. Prevalence of diabetes among men and women in China. N Engl J Med. 2010;362(12):1090-1101.

2. Xu Y, Wang L, He J, et al; 2010 China Noncommunicable Disease Surveillance Group. Prevalence and control of diabetes in Chinese adults. JAMA. 2013;310(9):948-959.

3. Pan X. Strive to prevent and control diabetes in China. Chin Med $J$ (Engl). 1995;108(2):83-85.

4. Pan C. Study on exploring diabetes present characteristics information in China. Section Endocrinal Foreign Med Sci. 2002;22(3):135-138.

5. Carpenter DM, Fisher EB, Greene SB. Shortcomings in public and private insurance coverage of diabetes self-management education and support. Popul Health Manag. 2012;15(3):144-148.

6. Perry HB, Zulliger R, Rogers MM. Community health workers in low-, middle-, and high-income countries: an overview of their history, recent evolution, and current effectiveness. Annu Rev Public Health. 2014;35:399-421.

7. Fisher EB, Boothroyd RI, Coufal MM, et al. Peer support for selfmanagement of diabetes improved outcomes in international settings. Health Aff (Millwood). 2012;31(1):130-139.

8. Moskowitz D, Thom DH, Hessler D, Ghorob A, Bodenheimer T. Peer coaching to improve diabetes self-management: which patients benefit most? J Gen Intern Med. 2013;28(7):938-942.

9. Thom DH, Ghorob A, Hessler D, De Vore D, Chen E, Bodenheimer TA. Impact of peer health coaching on glycemic control in lowincome patients with diabetes: a randomized controlled trial. Ann Fam Med. 2013;11(2):137-144.

10. Gagliardino JJ, Arrechea V, Assad D, et al. Type 2 diabetes patients educated by other patients perform at least as well as patients trained by professionals. Diabetes Metab Res Rev. 2013;29(2):152-160.
11. Chan JC, Sui Y, Oldenburg B, et al; JADE and PEARL Project Team. Effects of telephone-based peer support in patients with type 2 diabetes mellitus receiving integrated care: a randomized clinical trial. JAMA Intern Med. 2014;174(6):972-981.

12. Pérez-Escamilla R, Damio G, Chhabra J, et al. Impact of a community health workers-led structured program on blood glucose control among latinos with type 2 diabetes: the DIALBEST trial. Diabetes Care. 2015;38(2):197-205.

13. Collinsworth A, Vulimiri M, Snead C, Walton J. Community health workers in primary care practice: redesigning health care delivery systems to extend and improve diabetes care in underserved populations. Health Promot Pract. 2014;15(2)(Suppl):51S-61S.

14. Findley S, Matos S, Hicks A, Chang J, Reich D. Community health worker integration into the health care team accomplishes the triple aim in a patient-centered medical home: a Bronx tale. J Ambul Care Manage. 2014;37(1):82-91.

15. Mayer MK, Urlaub DM, Guzman-Corrales LM, Kowitt SD, Shea CM, Fisher EB. "They're doing something that actually no one else can do": a qualitative study of peer support and primary care integration. J Ambul Care Manage. In press.

16. Glasgow RE. RE-AIMing research for application: ways to improve evidence for family medicine. J Am Board Fam Med. 2006; 19(1):11-19.

17. Glasgow RE, Nelson CC, Strycker LA, King DK. Using RE-AIM metrics to evaluate diabetes self-management support interventions. Am J Prev Med. 2006;30(1):67-73.

18. Feldstein AC, Glasgow RE. A practical, robust implementation and sustainability model (PRISM) for integrating research findings into practice. Joint Commission journal on quality and patient safety / Joint Commission Resources. Apr 2008;34(4):228-243.

19. Fisher EB, Brownson CA, O'Toole ML, Anwuri VV. Ongoing follow-up and support for chronic disease management in the Robert Wood Johnson Foundation Diabetes Initiative. Diabetes Educ. 2007;33(Suppl 6):201S-207S.

20. Fisher EB, Earp JA, Maman S, Zolotor A. Cross-cultural and international adaptation of peer support for diabetes management. Fam Pract. 2010;27(Suppl 1):i6-i16.

21. World Health Organization. Definition, Diagnosis, and Classification of Diabetes Mellitus and its Complications: Report of a WHO Consultation. Geneva, Switzerland: World Health Organization; 1999.

22. Zhang L, Liu Z, Wang Z, Yi J, Wang Q. Diabetes Health Education and Effectiveness Evaluation. Chinese Journal of Prevention and Control of Chronic Non-Communicable Diseases. 1995;3(4):170-172.

23. Zeng Q, He Y, Dong S, et al. Optimal cut-off values of BMI, waist circumference and waist:height ratio for defining obesity in Chinese adults. Br J Nutr. 2014;112(10):1735-1744.

24. Bandura A. Self-efficacy mechanism in human agency. Am Psychol. 1982;37(2):122-147.

25. Yip WCM, Hsiao WC, Chen W, Hu S, Ma J, Maynard A. Early appraisal of China's huge and complex health-care reforms. Lancet. 2012;379(9818):833-842.

26. Kim HS, Sherman DK, Taylor SE. Culture and social support. Am Psychol. 2008;63(6):518-526.

27. Fisher EB, Brownson CA, O'Toole ML, Shetty G, Anwuri VV, Glasgow RE. Ecologic approaches to self-management: the case of diabetes. Am J Pub Health. 2005;95(9):1523-1535.

28. Marrero DG, Ard J, Delamater AM, et al. Twenty-first century behavioral medicine: a context for empowering clinicians and patients with diabetes: a consensus report. Diabetes Care. 2013;36(2):463-470.

29. Boothroyd RI, Fisher EB. Peers for progress: promoting peer support for health around the world. Fam Pract. 2010;27(Suppl 1):i62-i68.

30. Chan JC, So WY, Yeung CY, et al.; SURE Study Group. Effects of structured versus usual care on renal endpoint in type 2 diabetes: the SURE study: a randomized multicenter translational study. Diabetes Care. 2009;32(6):977-982. 